\title{
Variable cognitive benefits in HAART-treated patients: are aging and low cholesterol linked?
}

This article was published in the following Dove Press journal:

Neurobehavioral HIV Medicine

13 March 2010

Number of times this article has been viewed

\author{
María Jose Míguez-Burbano' \\ John E Lewis² \\ Ximena Burbano 3 \\ Rhonda Rosenberg' \\ Joel Fishman ${ }^{4}$ \\ Deshratn Asthana ${ }^{2}$ \\ Karl Goodkin ${ }^{5}$ \\ Robert M Malow' \\ 'Department of Health Promotion \\ and Disease Prevention, Robert \\ Stempel College of Public Health and \\ Social Work and College of Medicine, \\ Florida International University, \\ Miami, FL, USA; '2Departments of \\ Psychiatry and Behavioral Sciences, \\ ${ }^{3}$ Epidemiology and Public Health, \\ and ${ }^{4}$ Radiology, University of Miami \\ Miller School of Medicine, Miami, FL, \\ USA; ${ }^{5}$ Department of Psychiatry and \\ Behavioral Neurosciences at Cedars- \\ Sinai Medical Center and Department \\ of Psychiatry and Biobehavioral \\ Sciences, David Geffen School of \\ Medicine, University of California at \\ Los Angeles, Los Angeles, CA, USA
}

Correspondence: María Jose

Míguez-Burbano

Department of Health Promotion and

Disease Prevention Robert Stempel

College of Public Health and Social Work

Florida International University, FL, USA

Tel +l 3053484517

$\mathrm{Fax}+\mathrm{I} 305348490$ I

Email mjmiguezII63@bellsouth.net
Objective: Since a sizeable portion of the brain is composed of cholesterol, which serves various vital functions, we investigated interrelationships among hypocholesterolemia (HypoCHL $<150 \mathrm{mg} / \mathrm{dL}$ ) and neurocognitive impairment for people living with HIV (PLWH). Methods: Fasting blood samples were obtained in 125 PLWH younger than 45 years of age (Group 1) and 40 PLWH older than 46 years old (Group 2) at baseline and after 24 weeks on highly active antiretroviral therapy (HAART). Participants with HypoCHL were compared with non-HypoCHL on learning/memory (California Verbal Learning Test $=$ CVLT) and global neurocognitive status (HIV Dementia Scale $=$ HDS).

Results: HypoCHL was prevalent in Group $1(35 \%)$ and Group $2(30 \%)$ and associated with lower CVLT $(P=0.03)$ and HDS $(P=0.02)$ scores. After HAART in Group 1 , non-HypoCHL subjects improved in every neurocognitive parameter, but progress was limited and non-significant in the HypoCHL subjects. Despite better adherence and lower viral loads $(P=0.05)$ exhibited by Group 2, the HypoCHL participants showed declines in HDS scores (-2.5) and total CVLT ( -0.86 words). After adjusting for relevant variables, HypoCHL subjects from Group 1 showed a three-fold increased risk for scoring in the dementia range $(P<0.01)$, and the risk was even higher for HypoCHL participants in Group $2(\mathrm{OR}=5.0,95 \%$ CI: $1.25-21.3, P=0.008)$.

Conclusions: The association of HypoCHL with limited HAART neurocognitive recovery and even a continuous decline indicates the importance of restoring lipid balance.

Keywords: lipids, metabolism, cognition, HIV-associated neurocognitive disorders, HAART, HIV/AIDS

\section{Introduction}

HIV-associated neurocognitive disorders (HAND) remain an important cause of neurological morbidity. ${ }^{1,2}$ Though the mechanisms mediating HAND are becoming clearer, complexities still need to be better understood in the pathophysiological process leading to neurodegenerative and neurocognitive dysfunction. ${ }^{1,2}$ Elucidating more precisely, the mechanisms and pathways underlying neuronal damage is critical in developing therapies for ameliorating this devastating condition. To attain this goal, one critical factor is to understand brain substrates. Cholesterol is an essential element in the central nervous system (CNS), constituting $80 \%$ of its structure. It is also involved in several essential CNS functions such as membrane trafficking, signal transduction, myelin formation, and synaptogenesis..$^{3-5}$ Lipid abnormalities are of longstanding clinical concern in treating HIV, ${ }^{6}$ particularly hypocholesterolemia (HypoCHL), which is a prevalent comorbidity of HIV, even in the early stages. Compared to the general population, people living with HIV (PLWH) suffer a greater prevalence (40\%) 
of HypoCHL. Even though the mechanisms causing the decrease in plasma cholesterol are unclear, direct effects of HIV on lipid metabolism and altered cytokine profiles have been implicated in producing lipid abnormalities.

Considering the above, the meagerness of research on the deleterious effects of low cholesterol on brain function in PLWH is surprising. Nevertheless, accumulating evidence from studies in the general population indicates that HypoCHL is closely related to neurological diseases. ${ }^{4,5,7-12}$ For example, in the Smith-Lemli-Opitz syndrome, inadequate cholesterol causes severe neurodegeneration and early death. ${ }^{7}$ Moreover, in vivo evidence points to an association between lowered brain cholesterol and Alzheimer's disease. ${ }^{4,5-11}$ Prevention trials, which have shown reductions in coronary heart disease by lowering serum cholesterol concentrations in middle-aged adults by diet, drugs, or both, have also reported an increase in deaths due to suicide or violence. ${ }^{7-11}$ These findings have been linked to lowering cholesterol beyond the normal range, which is consistent with our studies showing the deleterious impact of HypoCHL on mood in PLWH. ${ }^{12}$ These findings are limited, however, by tests being conducted during the pre-highly active antiretroviral therapy (HAART) era and with predominantly younger PLWH.

Also requiring further investigation, is precisely how aging affects neurocognitive function of PLWH. ${ }^{13}$ Given the increased prevalence of neurocognitive disorders and considering that cholesterol degrades with age, the risk of HAND in the aging population of PLWH is of particular concern. ${ }^{13}$ Controversy exists, however, regarding the potential confounding effect of aging on neurocognitive performance in PLWH. ${ }^{13-19}$ Also, side effects from antiretroviral drugs might accentuate natural aging symptoms, ${ }^{13}$ and little is known about the influence of cholesterol on neurocognitive functioning among older PLWH. Further limiting our knowledge is the frequent exclusion of older PLWH from research, because of the concern that they may bias the immune decline outcome. Nonetheless, both HypoCHL and cognitive impairments are frequently described in the aging population.

This study provides the first examination, to our knowledge, of the effect of cholesterol status on neuropsychological performance before and after HAART in young and old PLWH. Based on current knowledge and considering that HIV has increasingly become a chronic disease, we hypothesized that: 1) despite the use of HAART, which often increases lipids, HypoCHL may still be prevalent due to aging of PLWH and 2) the deleterious effects of altering a key component of myelin, such as cholesterol, which is essential for fast transmission speed in neurons, will become more evident, particularly in older individuals.

\section{Methods}

\section{Sampling}

PLWH aged 18 to 60 years, who were either being started on their HAART regimen or were receiving a new antiretroviral regimen for less than 6 weeks, were eligible to be enrolled in the study. The definition of HAART used for analyses was guided by the published recommendations by the Panel on Antiretroviral Guidelines for Adults and Adolescents of $2008 .{ }^{20}$ For the purpose of these analyses, participants were divided into two groups by age: Group 1 included PLWH younger than $\leq 45$ years of age and Group 2 consisted of midlife-to-older PWLH ( $>46$ years). Patients were excluded if they were non-ambulatory or if they had other conditions that might produce neuropsychiatric or immune/thymus compromise other than HIV (ie, CNS opportunistic infection, head injury with or without loss of consciousness, tumors, major psychiatric disease, developmental disorders, severe malnutrition, or autoimmune diseases). Participants were also excluded if they had a family history of dyslipidemia or were receiving lipid lowering interventions. The Institutional Review Board at the University of Miami approved the research protocol.

\section{Exposure: cholesterol assessment}

Fasting blood samples were drawn and sent to a clinical laboratory for analysis within 6 hours of being collected. No participant was acutely ill at the blood draw. Total cholesterol (TC), high-density lipoprotein (HDL), low-density lipoprotein (LDL), and triglyceride (TG) levels were measured by routine enzymatic methods (KonePro, Konelab). HypoCHL (level $<150 \mathrm{mg} / \mathrm{dL}$ ) was defined according to the US National Cholesterol Education Program guidelines. ${ }^{21}$

\section{Neurocognitive outcomes}

The National Institute of Mental Health and the National Institute of Neurological Diseases and Stroke have published nomenclature and research case definitions to guide the diagnosis of neurologic manifestations of HIV-1 infection. ${ }^{2}$ In clinical practice, their use has been limited due to the extensive, time-consuming neurocognitive battery requirements and the need for specialized personnel. For this study, two neurocognitive tests were selected among those recommended for neurocognitive assessments of PLWH, and testing was conducted by a trained technician. 
Since memory has been the most predominant neuropsychological area impaired among PLWH, particularly before the advent of HAART, we selected a memory test, the California Verbal Learning Test (CVLT), ${ }^{22}$ as a primary outcome. Because aging is associated with a decline in memory function, this test was relevant for the study. The CVLT is a multidimensional measure of verbal learning and memory that includes two word lists, each of which contains 16 shopping items. ${ }^{22}$ Items are distributed according to a Monday list and a Tuesday distracter list with scores reflecting the number of objects that an individual can recall. Participants are asked to recall the Monday list spontaneously, following semantic cues and after a 20-minute delay. Finally, participants are given a "yes/no" recognition test in which they are presented with the 16 target words embedded within a list of 28 non-target words. Scores are based on the number of words provided by the participant on the various trials and on the number of times they correctly identify a word from the target list. ${ }^{22}$ Monolingual Spanish-speaking adults were tested with a translated version of the CVLT. Bilingual participants were provided with the option of being tested with the English or the Spanish version. ${ }^{23-25}$

The HIV Dementia Scale (HDS) is a simple screening test to identify subjects who probably have dementia and who would then need to undergo a complete assessment to confirm the diagnosis. ${ }^{26}$ The HDS, with a sensitivity of $80 \%$ and a specificity of $91 \%$ to detect dementia, has been shown to be superior to other widely used rapid screening tests, such as the Mini-Mental State Examination, and has been previously used to monitor HAART effects on the CNS..$^{26,27}$ The HDS is comprised of four tasks that evaluate the domains of memory, attention, psychomotor speed, and construction. A cutoff score of 10 on the HDS was used to screen out PLWH who were neurocognitively compromised. ${ }^{22,26-28}$

\section{Control variables}

At baseline and at the 6-month follow-up, each participant was interviewed using standardized research questionnaires covering: sociodemographic information, drug use, alcohol use, and past and current medical history. Participants were questioned with AIDS-defining Centers for Disease Control and Prevention (CDC) criteria to establish HIV disease status. ${ }^{29}$ Hemoglobin and platelet measures were also available and included in the database. Adherence was calculated using both pharmacy records and a standardized antiretroviral adherence questionnaire (Aids Clinical Trial Group $=$ ACTG).$^{30}$ Once visit procedures were completed, medical chart and pharmacy records were abstracted, and participant information was validated.
Anthropometrics and dietary intake were obtained using standardized protocol and then computerized using a nutritional analysis program. We collected detailed information regarding intake (dietary and supplements) of antioxidants and vitamin B6. In addition, plasma levels of vitamin B12 and homocysteine were measured at each visit.

CD4 cell count per microliter and HIV RNA copies per milliliter were used as markers of HIV disease progression. Plasma HIV viral burden was quantified using the UltraSensitive Roche HIV-1 MONITOR Test, version 1.5 (Roche Diagnostics, Branchburg, New Jersey, USA), which was used to quantify HIV-1 RNA copy number in the range of 50 to 750,000 copies $/ \mathrm{mL}$. Measurements of HIV-1 RNA concentration were transformed to the logarithm base 10 ( $\log 10$ or ' $\log$ ') per $\mathrm{mL}$ for data analysis. The percentage and absolute numbers of $\mathrm{T}$ lymphocyte sub populations $\mathrm{CD}^{+}, \mathrm{CD}^{+}$, and $\mathrm{CD} 8^{+}$cell counts were determined by flow cytometry.

\section{Statistical analyses}

The data were analyzed using SPSS 15.0 software (SPSS Inc., Chicago, Illinois, USA), and $P$-values $<0.05$ were considered to be statistically significant. Descriptive statistical analyses were conducted and mean variable values were compared between participants with and without low cholesterol (HypoCHL and non-HypoCHL) using Student's $t$ test and one-way analysis of variance procedures. These comparisons reflected the hypotheses that participants with HypoCHL, even if adhering to a prescribed regimen, would show poorer neurocognitive performance compared to those with normal cholesterol levels. These procedures allowed the determination of covariates for inclusion in the univariate analyses and in the multivariate model (ie, age, race/ ethnicity, education level, stress, and depression). Correlations among the selected variables of interest were examined with Pearson's coefficients.

Based on the HIV literature, several variables with the potential for confounding the results were analyzed in the descriptive and final analyses, and included sociodemographics (age, gender, and education), CDC status, drug use (including illegal drugs, prescribed drugs, tobacco, and alcohol), anemia, body mass index (BMI), vitamin (use and plasma level), hyperhomocysteinemia, and adherence.

Univariate analyses were used to calculate odds ratios (OR) and 95\% confidence intervals (CI). Analyses were repeated while controlling for age, education, history of drug abuse, viral load, CD4 cell count, and CDC stage of HIV-1 infection. Finally, outcomes and observed covariates 
that were significantly associated with HAART responses (naïve $\mathrm{CD} 4$, thymus size, or $\operatorname{HDS} P<0.05$ ) in univariate analysis were then considered in a multivariate model. Nonsignificant variables $(P \geq 0.05)$ were removed, beginning with the least significant variables, until the final full model was determined. Longitudinal regression analyses included the baseline value of the dependent variable. Adjusted OR for dichotomous variables were calculated by logistic regression. Model statistics included adjusted OR, 95\% CI, and their corresponding $P$-values.

\section{Results}

\section{Study population characteristics}

The study population included 165 PLWH who had baseline and 6 month follow-up blood samples drawn, and did not receive cholesterol-lowering medication. The mean TC level of the sample was $173 \pm 43 \mathrm{mg} / \mathrm{dL}(52-324 \mathrm{mg} / \mathrm{dL})$ with HypoCHL present in $40 \%$ of the study participants at baseline. Of note, only $5 \%$ of the group had more than $250 \mathrm{mg} / \mathrm{dL}$ TC. Cholesterol levels were unrelated to triglycerides and malnutrition (Table 1).

After HAART, the mean TC values of the group increased, but not significantly, by $4 \mathrm{mg} / \mathrm{dL}$, mostly as a reflection of $\mathrm{LDL}(+3.4 \mathrm{mg} / \mathrm{dL})$ and less due to HDL $(+1.1 \mathrm{mg} / \mathrm{dL})$. As a result, HypoCHL was less prevalent and hypercholesterolemia slightly increased (33\% vs $40 \%$ at baseline $3 \%$ vs $5 \%, P=$ NS).

Table 1 depicts the baseline demographics, main clinical variables, and corresponding $P$-values of the study population by cholesterol groups. Subgroups were comparable in sociodemographic variables, dietary intake, anthropometrics, CD4 counts, prior exposure to antiretroviral, and comorbid conditions, such as diabetes and hypertension.

Table I Baseline sociodemographic information of HIV-infected participants by study groups

\begin{tabular}{|c|c|c|c|c|c|c|}
\hline Variable & $\begin{array}{l}\text { Hypo- } \\
\text { cholesterolemia } \\
n=65\end{array}$ & $\begin{array}{l}\text { Nonhypo- } \\
\text { cholesterolemia } \\
n=100\end{array}$ & $P$-value & $\begin{array}{l}\text { Younger } \\
\text { PLWH } \\
n=I 25\end{array}$ & $\begin{array}{l}\text { Older } \\
\text { PLWH } \\
\mathbf{n}=\mathbf{4 0}\end{array}$ & $P$-value \\
\hline$\overline{M e n}$ & $72 \%$ & $68 \%$ & NS & $72 \%$ & $70 \%$ & NS \\
\hline Women & $28 \%$ & $32 \%$ & & $28 \%$ & $30 \%$ & \\
\hline Black & $65 \%$ & $55 \%$ & & $53 \%$ & $75 \%$ & \\
\hline Hispanic & $33 \%$ & $29 \%$ & & $37 \%$ & $19 \%$ & 0.02 \\
\hline White & $0 \%$ & $16 \%$ & 0.001 & $10 \%$ & $6 \%$ & \\
\hline$<\$ 10,000$ & $89 \%$ & $88 \%$ & & $89 \%$ & $87 \%$ & NS \\
\hline$\$ 11,000-\$ 20,000$ & $9 \%$ & $10 \%$ & & $9 \%$ & $11 \%$ & \\
\hline$>\$ 20,000$ & $2 \%$ & $2 \%$ & 0.7 & $2 \%$ & $2 \%$ & \\
\hline Hazardous alcohol use & $34 \%$ & $28 \%$ & 0.08 & $59 \%$ & $51.5 \%$ & NS \\
\hline Nonhazardous use & $66 \%$ & $72 \%$ & & $41 \%$ & $48.5 \%$ & \\
\hline Tobacco & $81 \%$ & $80 \%$ & NS & $81 \%$ & $80 \%$ & NS \\
\hline No & $19 \%$ & $20 \%$ & & $19 \%$ & $20 \%$ & \\
\hline Albumin & $4.0 \pm 0.4$ & $4 . I \pm 0.5$ & NS & $4.0 \pm 0.2$ & $4.0 \pm 0.5$ & NS \\
\hline Body mass index & $26.2 \pm 6.2$ & $27.4 \pm 6.7$ & NS & $24.2 \pm 68$ & $27.2 \pm 6.8$ & 0.04 \\
\hline Educational level & $1 \mathrm{I} .4 \pm 2.1$ & $10.6 \pm 3$ & NS & $11.5 \pm 2.5$ & $11.3 \pm 2.6$ & NS \\
\hline Number of years & $9.7 \pm 6.6$ & $8.7 \pm 5.6$ & NS & $8.65 \pm 5.4$ & $9.73 \pm 6.9$ & NS \\
\hline \multicolumn{7}{|l|}{ Diagnosed with HIV } \\
\hline Nadir CD4 cell counts & $193 \pm 172$ & $277 \pm 178$ & NS & $233 \pm 156$ & $252 \pm 183$ & NS \\
\hline \multicolumn{7}{|l|}{ CDC clinical } \\
\hline Disease & $12 \%$ & $20 \%$ & & $19 \%$ & $9 \%$ & \\
\hline Stage A & $39 \%$ & $51 \%$ & & $46 \%$ & $50 \%$ & \\
\hline Stage B & $49 \%$ & $29 \%$ & 0.005 & $35 \%$ & $41 \%$ & NS \\
\hline \multicolumn{7}{|l|}{ Stage C } \\
\hline Plasma HIV load (log) & $4.5 \pm 1.2$ & $3.8 \pm 1.7$ & 0.006 & $4.4 \pm 1.4$ & $3.8 \pm 1.6$ & 0.03 \\
\hline Previously exposed to antiretrovirals & $78 \%$ & $75 \%$ & NS & $73 \%$ & $78 \%$ & NS \\
\hline Adherence & $85 \%$ & $90 \%$ & NS & $83 \%$ & $91 \%$ & NS \\
\hline
\end{tabular}

Notes: Values are mean \pm standard deviation or percentages. The data represent descriptive statistics and comparisons of baseline key variables between younger individuals and those midlife-to-older PLWH ( $>46$ years). Age subgroups were dissimilar in race/ethnicity. Older individuals exhibited better adherence. Although no significant differences in sociodemographic characteristics were found between cholesterol groups, they significantly differed in race and their viroimmune status.

Abbreviation: PLWH, people living with HIV. 


\section{Age and the baseline characteristics of the study population}

Given the potential effects of age on the study variables, Table 1 also illustrates the sociodemographic and clinical characteristics of the study population by age group. As already noted, Group 1 consisted of younger PLWH, $\leq 45$ years of age, and Group 2 were midlife to older age. The two age groups were comparable in most of the variables, however, we noted two exceptions at baseline: participants with HypoCHL were less likely to be white (95\% CI: $0.0-0.48$; $P=0.001)$ and to have lower CD4 cell counts and higher viral loads. Age subgroups were dissimilar in race/ethnicity. Individuals in Group 2 were three times more likely to be African American (OR = 2.7, 95\% CI: 1.3-6.0; $P=0.004)$ and less likely to be Hispanic (OR $=0.5,95 \% \mathrm{CI}$ : $0.3-0.9$; $P=0.02)$. As expected, Group 2 had greater odds of having diabetes and/or hypertension $(\mathrm{OR}=1.8,95 \% \mathrm{CI}$ : 1.2-2.5; $P=0.01)$. Although participants in Group 2 exhibited a lower (BMI), this was not clinically relevant, since albumin levels and proportion of malnutrition (albumin $<3.5$ ) were similar between groups. As a continuous outcome, the adjusted percent adherence difference between the two groups was only $8 \%(P=0.1)$ with better adherence exhibited by older individuals.

Mean (SD) TC of Group 1 was $172 \pm 43.6 \mathrm{mg} / \mathrm{dL}$ and slightly lower, $164 \pm 36 \mathrm{mg} / \mathrm{dL}$, in Group 2. In contrast, the non-HypoCHL category was predominantly comprised of those with normal cholesterol values $(95 \% \mathrm{CI}$ $151-200 \mathrm{mg} / \mathrm{dL}$ ) with only $5 \%$ fulfilling hypercholesterolemia criteria. Both Group 1 and 2 had a sizable proportion who met HypoCHL criteria (G2 = 41\% vs G1 = 37\%) and more men than women $(\mathrm{OR}=2.9, P=0.09)$ exhibited HypoChl. Of interest, analyses after HAART indicated that lipid changes varied by age group. Participants in Group 2 evidenced drops in both TC and LDL $(-5 \mathrm{mg} / \mathrm{dL}$ and $-4 \mathrm{mg} / \mathrm{dL}$, respectively) and increases in $\mathrm{HDL}$ (+1 mg/dL). In contrast, Group 1 showed non-significant increases in TC, LDL, and HDL $(+5.3 \mathrm{mg} / \mathrm{dL},+4.4 \mathrm{mg} / \mathrm{dL}$, and $+1.1 \mathrm{mg} / \mathrm{dL}$, respectively).

Since we have previously shown that liquor (ie, rum, whisky, vodka) has more severe adverse effects than other types of alcohol (beer or wine), we explored the potential effect of specific alcoholic beverages, while controlling for age. ${ }^{31}$ Compared to non-liquor users, liquor users had significantly lower LDL $(78 \pm 30$ vs $93 \pm 34 \mathrm{mg} / \mathrm{dL} ; P=0.008)$, but similar HDL values $(48.8 \pm 15$ vs $47.5 \pm 15.2 ; P=0.5)$ Wine displayed opposite associations (higher HDL levels: $46.5 \pm 13$ vs $42.7 \pm 12.3 \mathrm{mg} / \mathrm{dL} ; P=0.04$; LDL: $87.8 \pm 35 \mathrm{vs}$
$80.8 \pm 31 \mathrm{mg} / \mathrm{dL} ; P=0.3)$. Individuals in Group 1 with HypoCHL were more likely to be hazardous liquor users than non-HypoCHL youths $(\mathrm{OR}=2.2,95 \% \mathrm{CI}$ : $1-5.3$; $P=0.05)$. However, the results were not significant, when the same analyses were performed with Group 2.

\section{Baseline neurocognitive performance by study subgroups}

The proportion of PLWH in both groups that scored below normal range in the HDS was similar. Briefly, $39 \%$ of Group 2 and $42 \%$ of Group 1 scored in the range suggestive of dementia. No significant correlations were found between the HDS and sociodemographic variables, including education, with the single exception of gender. No significant racial differences in mean HDS were evident. Among Group 1, men had a higher tendency to score $\leqslant 10$ than women $(\mathrm{OR}=2.5$, $P=0.05)$.

To determine whether HypoCHL was functionally relevant, we compared neurocognitive performance at baseline and, again, after 6 months of HAART between the cholesterol groups. A significant correlation was observed between cholesterol and mental status, as indicated by baseline total HDS $(\mathrm{r}=0.19, P=0.05)$. Those with HypoCHL scored significantly lower on the HDS $(8.9 \pm 3.7$ vs $10.4 \pm 3.5 ; P=0.02$ ). Moreover, Group 1 individuals with HypoCHL were 3 times more likely to score in the dementia range (prevalent dementia) than those with nonHypoCHL (OR $=2.75,95 \% \mathrm{CI}: 1.14-6.47 ; P=0.001)$. Conversely, no participants scoring in the dementia range had hypercholesterolemia.

Compared to non-HypoCHL, PLWH with HypoCHL performed worse (greater impairment) on the CVLT, especially in recall words from the Monday list $(7.1 \pm 3.7$ vs $9 \pm 3.5$; $P=0.02)$, Tuesday list (5.0 \pm 2.0 vs $6.24 \pm 2$ words; $P=0.01)$, long delay recall $(8.9 \pm 3.7$ vs $10.4 \pm 3.5 ; P=0.04)$, and total CVLT score $(9.8 \pm 3.3$ vs $11.1 \pm 2.9$ words; $P=0.03)$.

Finally, to evaluate the influence of these demographic variables on CVLT performance, a multiple regression analysis was performed using race, education, and gender as independent variables. The analysis indicated that none of these demographic characteristics significantly $(P<0.05)$ influenced CVLT performance for this group of PLWH, although gender had a borderline effect in some CVLT indices ( $P=0.06$ and 0.08 , respectively).

A tendency was also observed for Caucasians and African Americans to outperform Hispanics in several individual and the total score $(P=0.08)$. In light of these findings we pursued analyses using both the raw and the T scores. 


\section{Longitudinal analyses of neurocognitive performance after HAART}

To further examine how cholesterol status might distinctively affect neurocognitive-related HAART effectiveness by age, improvements after HAART were measured and compared between the 4 study subgroups (Table 2). Group 1 individuals, with normal cholesterol levels, improved significantly on the HDS $(+1.2, P=0.09)$, the total CVLT (raw score $=+1.5$ words, $P=0.0001$; T score $=+15, P=0.001$ ), and the long-delayed scores $(+2.7$ words, $P=0.0001$; T score $=+8, P=0.01$ ). Significant improvements in the younger subgroup with HypoCHL were limited to the long-delayed scores ( +2 words, $P=0.03$; T score $=-4.5$, $P=0.1$ ). Among Group 2, the subgroup with HypoCHL was characterized by a decline on the HDS ( -3 points) and the total CVLT scores $(-1$ words; T score $=-15, P=0.001)$ and only tended to improve in long-delayed scores $(+0.97$, $P=0.08$; T score $=+1.8, P=0.3)$. Conversely, the older PLWH with non-HypoCHL tended to maintain their scores $($ CVLT $=+0.7$; T score $=+1.5, P=0.3$ and long-delayed $=$ +0.6 ; $\mathrm{T}$ score $=+0.8, P=0.6$ and verbal memory item on the HDS $=-0.5$ words).

Analyses were repeated for participants with a CD4 cell count $>200$ cells to assess the potential effects of immune status on study outcomes. As illustrated in Table 3, all the results are similar indicating that HypoCHL is not the consequence of an advanced stage of the disease, but rather it appears to be an early event that continues through different stages of the disease and is relevant for those on HAART.

With regards to gender, at follow-up, young men were 3 times more likely to score in the dementia range (prevalent dementia) than young women ( $\mathrm{OR}=3.2,95 \% \mathrm{CI}: 1.16-9.4$; $P=0.02$ ). No such tendency was observed in the older group in which men and women had similar risks. No significant racial/ethnic differences in cognitive improvements were evident.

\section{Incident neurocognitive impairment}

A total of 99 subjects were neurocognitively normal at their baseline assessment. Of these, 37\% subsequently scored within the range associated with dementia. The decline in HDS levels was strongest and most significant in those with baseline HypoCHL ( -3.5 points, $P=0.05$ ), as opposed to those with normal baseline cholesterol levels $(+0.7$, $P=0.4)$. Noteworthy is that no significant difference in incident impairment was evident between Groups 1 and 2 (35\% vs $46 \%$ ). After adjusting for age, race, sex, education, adherence, and baseline virologic and immunologic status, data analyses revealed a significant association of HypoCHL with incident neurocognitive impairment (defined as HDS $\leq 10$ at the last visit, $\mathrm{OR}=2.11,95 \%$ CI: $1.303-4.3 ; P=0.03$ ).

\section{Regression analyses}

To reveal the independent determinants of scoring in the dementia range, stepwise multiple linear regression analyses were performed with HDS less than 10 as the dependent variable in each model. TC groups, baseline CDC clinical stage, plasma viral load, adherence, age category (by 10 years), gender, alcohol, education, hyperhomocysteinemia, and drug use were considered as independent variables. After adjusting for these variables, young individuals with HypoCHL showed a three-fold increased risk for scoring in

Table 2 Cognitive changes after HAART in PLWH with more than 200 CD4 cell counts

\begin{tabular}{|c|c|c|c|c|c|c|c|c|}
\hline Variables & $\begin{array}{l}\text { Younger } \\
\text { HypoCHL } \\
n=24\end{array}$ & $P$-value & $\begin{array}{l}\text { Younger } \\
\text { non-HypoCHL } \\
n=35\end{array}$ & $P$-value & $\begin{array}{l}\text { Older } \\
\text { HypoCHL } \\
\mathbf{n}=\mathbf{8}\end{array}$ & $P$-value & $\begin{array}{l}\text { Older } \\
\text { non- } \\
\text { HypoCHL } \\
n=10\end{array}$ & $P$-value \\
\hline Tuesday & $0.7+0.6$ & 0.3 & $I . I+0.4$ & 0.006 & $-0.5+0$ & I & $1.1+0.3$ & 0.007 \\
\hline Monday Recall & $1.7+0.9$ & 0.8 & $2.2+0.9$ & 0.02 & $-1+0.95$ & 0.4 & $0.5+0.3$ & 0.5 \\
\hline Long-Delay Recall & $0.8+1$ & 0.1 & $2.1+0.6$ & 0.002 & $-0.8+0.7$ & 0.4 & $0.5+0.4$ & 0.3 \\
\hline Total scores & $1.9+0.3$ & 0.02 & $2.6+1$ & 0.02 & $-1.7+0.6$ & 0.07 & $0.9+0.4$ & 0.07 \\
\hline $\begin{array}{l}\text { HIV dementia } \\
\text { Scale }\end{array}$ & $0.6+0.4$ & 0.7 & $0.8+0.6$ & 0.2 & $-3+1.8$ & 0.01 & $1.9+1.1$ & 0.1 \\
\hline
\end{tabular}

Notes: This table illustrates changes in cognitive parameters in 77 (only those patients with more than $200 \mathrm{CD} 4$ cell counts) PLWH according to age and cholesterol group. The $P$-values represent paired $t$-tests comparing the groups mean variables at baseline and after 24 weeks on HAART. Statistically significant values $(P<0.05)$ are in bold print and are mostly associated with the younger non-HypoCHL subgroup. Negative values indicated a decline in the mean score. The association of $\mathrm{HypoCHL}$ (gray cells) with either limited improvement or sustained impairment suggests that restoring cholesterol balance increases the likelihood of neurocognitive recovery with HAART. Abbreviations: HAART, highly active antiretroviral therapy; PLWH, people living with HIV; HypoCHL, hypocholesterolemia. 
the dementia range $(\mathrm{OR}=3.2,95 \% \mathrm{CI}: 1.34-7.62 ; P=0.01)$ compared to their counterparts with non-HypoCHL (Table 3 ). Failing to achieve undetectable plasma viral loads and liquor use (OR $=6.6,95 \% \mathrm{CI}: 2.3-18 ; P=0.005)$, but not wine use, were the only other variables identified as significant risk factors for onset or worsening of HDS. The risk was even higher for Group 2 individuals with HypoCHL (OR = 4.6, 95\% CI: $1.3-16.3 ; P=0.02)$. Among Group 2, failing to achieve undetectable plasma viral loads, low hemoglobin levels, BMI, and CD4 cell counts $<200$ cells were not associated with progression of neurocognitive deficits. Since the stratified analyses for these points were indicative of meaningful differences between subgroups, the CI estimates were wide and overlapping. Therefore, a formal test of interaction was assessed, but was not statistically significant $(P=0.08)$.

\section{Discussion}

Despite a general belief that HypoCHL will no longer be a relevant issue in the HAART era, our results indicated that HypoCHL is still prominent in PLWH and, in fact, is more prevalent than hypercholesterolemia. Our findings also demonstrate that less-than-expected neurocognitive improvement in antiretroviral-treated individuals is associated with a high prevalence of HypoCHL in all age groups, particularly in the older individuals. Although, to the best of our knowledge, this represents the first paper examining HypoCHL's neurocognitive implications in relationship to HAART, this is not the first time that low cholesterol has been linked with a neurological disease. ${ }^{7,9,32-36}$ Longitudinal studies in the general population have found an association between low total cholesterol and poor neurocognitive performance. ${ }^{33}$ Moreover, Romas et $\mathrm{al}^{34}$ found that subjects with the lowest $(<177 \mathrm{mg} / \mathrm{dL}[<4.6 \mathrm{mmol} / \mathrm{L}])$ baseline TC level had an increased risk for incident dementia after 2.5 years of follow-up. Treatments to correct HypoCHL (ie, dietary

Table 3 Final model odds ratios for neuropsychological impairment by age group

\begin{tabular}{lllllll}
\hline Model & $\begin{array}{l}\text { Young } \\
\text { PLWH } \\
\text { OR }\end{array}$ & 95\% Cl & P-value & $\begin{array}{l}\text { OLD } \\
\text { PLWH } \\
\text { OR }\end{array}$ & 95\% Cl & P-value \\
\hline $\begin{array}{l}\text { Hypocholester- } \\
\text { olemia }\end{array}$ & 3.2 & $1.3-7.6$ & 0.01 & 4.6 & $1.3-16.3$ & 0.02 \\
$\begin{array}{l}\text { Detectable } \\
\text { viral loads } \\
\text { after HAART }\end{array}$ & 6 & $2.3-18$ & 0.005 & 1.1 & $0.15-7.4$ & 0.9 \\
\begin{tabular}{l} 
Liquor use \\
\hline
\end{tabular} & 1.8 & $1.1-3.7$ & 0.04 & 1.3 & $0.9-2.2$ & 0.1 \\
\hline
\end{tabular}

Abbreviations: PLWH, People living with HIV; OR, odds ratios; $\mathrm{Cl}$, confidence intervals. intervention or supplements) may or may not be of some clinical benefit for PLWH if the current findings are substantiated by a much larger longitudinal study evaluating if HypoCHL is a cause or an effect of the underlying process.

Although vascular problems may account for a positive correlation between total cholesterol level and neurocognitive impairment at the high end of the scale, several nonvascular factors may mediate such a relationship at the low end of the scale as well. ${ }^{35}$ First, we have previously demonstrated that HypoCHL induced functional alterations in CD8 cells, which have been associated with impaired viral clearance. ${ }^{31}$ Second, in accord with previous studies; achieving undetectable plasma viral load has been a protective factor against neurocognitive impairment. In this regard, it needs to be highlighted that our HypoCHL subjects exhibited higher plasma viral loads. In addition, retroviruses use several different mechanisms to traverse the blood-brain barrier, such as via macropinocytosis, which is dependent on intact lipid rafts (cholesterol-rich patches). Third, it is important to underscore that liquor consumption in our study population was a risk factor for both HypoCHL and neurocognitive impairment. Our results are consistent with other studies in the general population showing that liquor and wine displayed opposite associations with dementia. ${ }^{36}$ Finally, considering that cholesterol depletion may also alter the availability of neurotransmitters, it is not surprising that our HypoCHL participants performed poorly and exhibited neurocognitive declines.

Our results concur with studies of samples without HIV infections, indicating the different ways that brain damage may be manifested across the lifespan. HypoCHL was not merely more prevalent in the older group; our findings suggest that their brains are more susceptible to such deleterious changes. The fact that baseline HypoCHL predicted treatment responses and that HypoCHL precedes the onset of dementia in some of these individuals, suggests, in fact, that low cholesterol is not an epiphenomenon but a possible contributing factor to the mechanism underlying this association. These findings are of great relevance considering that in the USA, as well as in other countries, more than half of the PLWH will be over 50 years of age by $2015 .{ }^{37}$ Also, even though older subjects were more likely to have low plasma cholesterol levels, it is notable that a significant proportion of young individuals had HypoCHL, and thus they may be potentially much more compromised in their future neurocognitive resilience. Our findings may consequently be relevant to the entire population of individuals living with 
HIV and therefore we believe that PLWH should be given information about the risk of HypoCHL.

Potential confounders, sporadically associated with a higher occurrence of dementia and neurocognitive impairment in PLWH, such as nutritional status and co-morbidities, were carefully analyzed. Even though older participants exhibited a lower BMI, the association of HypoCHL with neurocognitive changes was observed mostly in those with normal BMI, and neither BMI, albumin levels, nor antioxidants was significant in the final model. Furthermore, the presence of comorbid conditions, such as diabetes, hypertension, anemia, or drug abuse cannot explain the lack of neurocognitive improvement nor even the continuous decline exhibited by this group, since no significant differences were detected on these indicators.

Nonetheless, our study has certain limitations. For example, participants were recruited in a primary public care setting. Thus, it is possible that our results are specific to this group, although we believe this to be unlikely. In addition, in order to be practical for this clinical setting the neurocognitive assessment was brief, indicating that additional studies with more complete batteries and longer follow-ups will be key in confirming this study's findings. While our translated version of the CVLT needs additional refinement, our finding that Spanish speakers tended to performed worse in the CVLT regardless of the version they used (standard English version or the translated version that requires further refinement), raises two questions: 1) How suitable are these norms are for Hispanic adults? 2) How appropriate is it to lump Hispanics and Caucasians together when using the corrected T scores? Bearing these questions in mind, one of the co-authors is pioneering the generation of a normative cognitive battery for the growing population of Hispanics living with HIV. ${ }^{23-25}$ Finally, the invasive nature of experiments that can determine brain lipid changes has limited this study to the investigation of plasma cholesterol levels. Nevertheless, previous studies in individuals with neuropsychological disorders have demonstrated correlations between peripheral measurements and lipid brain abnormalities.

Despite these limitations, the present study is unique in several aspects. It is the first to examine, prospectively, the association between HypoCHL and HAART neurocognitive responses. Our study is longitudinal, rather than crosssectional. Moreover, our data suggest that HypoCHL may be a marker for early processes that reflect neurodegenerative changes and thus may be of great clinical value. Additional studies to explore this finding could be instructive.

The healthy utilization of cholesterol by the body is increasingly found to be essential to the function of different organ systems, overall longevity, and quality of life. Efforts to remedy disruptions in the body's healthy use of cholesterol are not straightforward. In the presence of HIV infection, the clinical picture is further complicated. As stated by more and more researchers currently on the trail of characterizing the intricate relationship between the body's lipid metabolism and HIV, cholesterol is not only essential to a healthy human organism, but also to the pathogenesis of HIV within the human host. ${ }^{38-42}$ Our findings indicate the need for increasing our collective understanding of how HIV commandeers the use of cholesterol in the body, and underscores the urgency of bringing the brain's utilization of cholesterol into the equation of HIV prevention.

\section{Acknowledgments}

The study was funded and analyzed by the NIAAA of the United States (5R21AA13793-3 and 3R01AA017405-02S1 MJM).

\section{Disclosures}

The authors report no conflicts of interest in this work.

\section{References}

1. Minagar A, Commins D, Alexander JS, et al. NeuroAIDS: characteristics and diagnosis of the neurological complications of AIDS.(Neurological Disorders). Mol Diagn Ther. 2008;12(1):25-43.

2. Antinori A, Arendt G, Becker JT, et al. Updated research nosology for HIVassociated neurocognitive disorders. Neurology. 2007;69:1789-1799.

3. Valenza M, Cattaneo E. Cholesterol dysfunction in neurodegenerative diseases: is Huntington's disease in the list? Prog Neurobiol. 2006;80(4):165-176.

4. Merched A, Xia Y, Visvikis S, Serot JM, Siest G. Decreased highdensity lipoprotein cholesterol and serum apolipoprotein A-I concentrations are highly correlated with the severity of Alzheimer's disease. Neurobiol Aging. 2000;21:27-30.

5. Park IH, Hwang E, Hong $\mathrm{H}$, et al. Lovastatin enhances Abeta production and senile plaque deposition in female Tg2576 mice. Neurobiol Aging. 2003;24:637-643.

6. Crook M. The basis and management of metabolic abnormalities associated with cardiovascular risk in human immunodeficiency virus infection and its treatment. Ann Clin Biochem. 2007;44:219-231.

7. Porter FD. RSH/Smith-Lemli-Opitz syndrome: a multiple congenital anomaly/mental retardation syndrome due to an inborn error of cholesterol biosynthesis. Mol Genet Metab. 2000;71:163-174.

8. Reitz C, Tang MX, Luchsinger J, Mayeux R. Relation of plasma lipids to Alzheimer disease and vascular dementia. Arch Neurol. 2004;61:705-714.

9. Mielke MM, Zandi PP, Sjogren M, et al. High total cholesterol levels in late life associated with a reduced risk of dementia. Neurology. 2005;64:1689-1695.

10. Kuusisto J, Koivisto K, Mykkänen L, et al. Association between features of the insulin resistance syndrome and Alzheimer's disease independently of apolipoprotein E4 phenotype: cross sectional population based study. BMJ. 1997;315:1045-049.

11. Scacchi R, De Bernardini L, Mantuano E, et al. DNA polymorphisms of apolipoprotein B and angiotensin I-converting enzyme genes and relationships with lipid levels in Italian patients with vascular dementia or Alzheimer's disease. Dement Geriatr Cogn Disord. 1998; 9(4):186-190. 
12. Shor-Posner G, Feaster D, Baldewicz T, Blaney, NT, et al. Psychological distress in HIV-1 disease in relationship to hypocholesterolemia. Int'l J of Psychiat in Med. 1997;27(2):159-171.

13. Valcour V, Shikuma C, Shiramizu B, et al. Higher frequency of dementia in older HIV-1 individuals: the hawaii aging with HIV-1 cohort. Neurology. 2004;63:822-827.

14. Goodkin K, Wilkie FL, Concha M, et al. Aging and neuro-AIDS conditions: a potential interaction with the changing spectrum of HIV-1 associated morbidity and mortality in the era of HAART? J Clin Epidemiol. 2001;54:S35-S43.

15. Goodkin K, Concha M, Jamieson BD, et al. Interaction of the aging process with neurobehavioral and neuro-AIDS conditions in the HAART era. In: Goodkin K, Shapshak P, Verma A, editors. Neuro-AIDS in the HAART Era: Pathophysiology, Diagnosis and Treatment. Washington, DC: American Society for Microbiology Press; 2009:473-486.

16. Pereda M, Ayuso-Mateos JL, Gomez Del Barrio A, et al. Factors associated with neuropsychological performance in HIV-seropositive subjects without AIDS. Psychol Med. 2000;30:205-217.

17. Van Gorp WG, Miller EN, Marcotte TD, et al. The relationship between age and cognitive impairment in HIV-1 infection: findings from the multicenter AIDS cohort study and a clinical cohort. Neurol. 1994:44:929-935.

18. Kissel E, Pukay-Martin N, Bornstein R. The relationship between age and cognitive function in HIV-infected men. J Neuropsychiatry Clin Neurosci. 2005;17:180-184.

19. Vance D, Ross L, Downs Ch. Self-reported cognitive ability and global cognitive performance in adults with HIV. The Journal of Neuroscience Nursing. 2008 May. http://www.medscape.com/viewarticle/571448 Accessed July 15, 2009.

20. DHHS/Henry J Kaiser Family Foundation Panel on clinical practices for the treatment of HIV infection. Guidelines for the use of antiretroviral agents in HIV-infected adults and adolescents. Updated May 2006. Available from: http://aidsinfo.nih.gov/ContentFiles/ AdultandAdolescentGL05042006050.pdf. Accessed July 10, 2009.

21. Grundy SM, Cleeman JI, Merz CN, et al. US national cholesterol guidelines implications of recent clinical trials for the national cholesterol education program adult treatment panel III guidelines. Coordinating Committee of the National Cholesterol Education Program; National Heart, Lung, and Blood Institute; American College of Cardiology Foundation; American Heart Association. Arterioscler Thromb Vasc Biol. 2004;24:e149-e161.

22. Delis DC, Kramer JH, Kaplan E, Ober BA. California Verbal Learning Test, New York, NY: The Psychological Corporation; 1987.

23. Goodkin K, Hardy DJ, Lopez E, et al. Performance of screening tests for HIV-associated neurocognitive disorder risk in an Argentinean sample. In: Abstracts of the Third NIMH Meeting on HIV Infection and the Central Nervous System. Stresa - Lago Maggiore, Italy, October, 22-24, 2009

24. Goodkin K, Cahn P, Concha C, Abusamra L, Lopez E, Kogan J. Prevalence of HIV-1-associated neurocognitive disorders in Argentina. In: Abstracts of the 5TH IAS Conference on HIV Pathogenesis, Treatment and Prevention July 19-22, 2009, Cape Town, South Africa, \#MOPEB028.
25. Lopez E, Morales G, and Goodkin K. "Hispano": Una consideracion del termino para trastornos cognitivos en VIH. (Spanish). Actualizaciones en SIDA. 2009;17(65):95-103.

26. Power C, Selnes OA, Grim JA, McArthur JC. The HIV Dementia Scale: A rapid screening test. J AIDS. 1995;8:273-278.

27. Dougherty RH, Skolasky RL, McArthur JC. Progression of HIVassociated dementia treated with HAART. AIDS Read. 2002;12: 69-74.

28. Smith CA, van Gorp WG, Ryan ER, et al. Screening subtle HIV-related cognitive dysfunction: The clinical utility of the HIV Dementia Scale. J Acquir Immune Defic Syndr. 2003;33:116-118.

29. Centers for Disease Control and Prevention. 1993 revised classification system for HIV infection and expanded surveillance case definition for AIDS among adolescents and adults. MMWR Recomm Rep. 1992 Dec 18;41(RR-17):1-19. Available online at www.cdc.gov/mmwr/preview/ mmwrhtml/00018871.htm. Accessed June 29, 2009.

30. Global Health Delivery Project. ACTG adherence baseline and followup questionnaires. May 2009. Available from http://www.ghdonline. org/adherence/resource/actg-adherence-baseline-and-follow-upquestionnair/. Accessed July 13, 2009.

31. Míguez-Burbano MJ, Lewis JE, Fishman J, et al. The influence of different types of alcoholic beverages on disrupting HAART. Alcohol Alcohol. 2009;44(4):366-371.

32. Stewart R, White L, Xue Q, Launer L. Twenty-six-year change in total cholesterol levels and incident dementia: The Honolulu-Asia aging study. Arch Neurol. 2007;64:103-107.

33. Penelope E, Elias M, D’Agostino R, Sullivan L, Wolf P. Serum cholesterol and cognitive performance in the Framingham Heart Study. Psychosom Med. 2005;67:24-30.

34. Romas SN, Tang MX, Berglund L, Mayeux R. APOE genotype, plasma lipids, lipoproteins, and AD in community elderly. Neurology. 1999;53:517-521.

35. Panzaa F, Solfrizzia V, D'Intronoa A, et al. Higher total cholesterol, cognitive decline, and dementia. Neurobiol Aging. 2009;30(4): 546-548.

36. Barefoot J, Grønbæk M, Feaganes J, et al Alcoholic beverage preference, diet, and health habits in the UNC Alumni Heart Study. Am J Clin Nutr. 2002;76:466-472.

37. Stoff DM, Khalsa JH, Monjan A, Portegies P. Introduction: HIV/AIDS and aging. Aids. 2004;18 Suppl 1:S1-S2.

38. Mujawar Z, Rose H, Morrow MP, Pushkarsky T, Dubrovsky L, et al. Human immunodeficiency virus impairs reverse cholesterol transport from macrophages. PLoS Biol. 2006;4(11):e365.

39. Gross L. HIV-Cholesterol connection suggests a new antiretroviral strategy. PLoS Biol. 2006;4(11):e375-e400.

40. Verma SP. HIV: a raft-targeting approach for prevention and therapy using plant derived compounds (review). Curr Drug Targets. 2009; 10(1):51-59.

41. Tang Y, Leao IC, Coleman EM, Broughton RS, Hildreth JE. Deficiency of Niemann-Pick type C-1 protein impairs HIV-1 release and results in gag accumulation in late endosomal/lysosomal compartments. J Virol. 2009;83(16):7982-7995.

42. Rose H, Hoy J, Woolley I, et al. HIV infection and high density lipoprotein metabolism. Atherosclerosis. 2008;199(1):79-86.

Neurobehavioral HIV Medicine

\section{Publish your work in this journal}

Neurobehavioral HIV Medicine is an international, peerreviewed, open access journal focusing on advances in research in HIV/AIDS, with specific reference to the neurological, psychiatric and behavioral consequences of the disease, concomitant infections and specific antiretroviral therapy. The manuscript

management system is completely online and includes a very quick and fair peer-review system, which is all easy to use. Visit http://www.dovepress.com/testimonials.php to read real quotes from published authors. 